\title{
Random Schrödinger operators with a background potential
}

\author{
Hayk Asatryan and Werner Kirsch \\ Faculty of Mathematics and Computer Science \\ FernUniversität in Hagen, Germany
}

April 10, 2019

\begin{abstract}
We consider one-dimensional random Schrödinger operators with a background potential, arising in the inverse problem of scattering. We study the influence of the background potential on the essential spectrum of the random Schrödinger operator and obtain Anderson Localization for a larger class of one-dimensional Schrödinger operators. Further, we prove the existence of the integrated density of states and give a formula for it.
\end{abstract}

\section{Introduction}

The fundamental work of physicist P. W. Anderson [2] gave rise to a lot of further investigations of different mathematicians. P. W. Anderson argued that electrons inside a disordered quantum mechanical system are localized (named Anderson Localization lately), provided that the degree of randomness of the impurities or defects is sufficiently large. One way to express Anderson Localization in mathematically rigorous terms is that the corresponding Schrödinger (Hamilton) operator has a pure point spectrum. For one-dimensional Schrödinger operators Anderson expected localization for all energies and arbitrary small disorder.

In the one-dimensional model the Schrödinger operator is the alloy-type operator

$$
-\frac{d^{2}}{d x^{2}}+V_{\omega}
$$

where the random potential $V_{\omega}$ may for example be of the form

$$
V_{\omega}(x):=\sum_{k=-\infty}^{\infty} q_{k}(\omega) f(x-k) \quad(x \in \mathbb{R})
$$

Here $f$ is a real-valued function and $q_{k}(k \in \mathbb{Z})$ are independent random variables with a common 
distribution $P_{0}$. The first proof of Anderson Localization for a related one-dimensional model was given by I. Goldsheid, S. Molchanov and L. Pastur in [8]. For the discrete analogue of (1) localization was first proved by H. Kunz and B. Souillard [12]. The first complete proofs of localization in the higher dimensional case were given in [7], [1]. The existence of the integrated density of states for alloy-type operators was established in [13] and [11].

On the space $L^{2}(\mathbb{R})$ we consider Schrödinger operators of the form

$$
H_{\omega}:=-\frac{d^{2}}{d x^{2}}+U+V_{\text {per }}+V_{\omega}
$$

We assume that the background potential $U$ belongs to the space of real-valued uniformly locally square-integrable functions

$$
L_{\text {unif }}^{2, \text { loc }}:=\left\{F:\left.\mathbb{R} \rightarrow \mathbb{R}\left|\sup _{x \in \mathbb{R}} \int_{x-1}^{x+1}\right| F(x)\right|^{2} d x<\infty\right\}
$$

and satisfies the relations

$$
U(x) \rightarrow a^{-} \quad \text { as } \quad x \rightarrow-\infty, \quad U(x) \rightarrow a^{+} \quad \text { as } \quad x \rightarrow+\infty
$$

with $a^{ \pm} \in \mathbb{R}$. The potential $U$ arises in the inverse problem of scattering (see [3]). $V_{\text {per }}$ is assumed to be a 1-periodic real-valued function in $L_{\text {unif }}^{2, \text { loc }}$, and $V_{\omega}$ is a random alloy-type potential of the form (1). We suppose that $f$, called the single-site potential, satisfies the estimate

$$
|f(x)| \leqslant C(1+|x|)^{-\gamma} \quad(x \in \mathbb{R})
$$

for some $\gamma>1$.

We assume for simplicity that $\operatorname{supp} P_{0}$ is a compact subset of $\mathbb{R}$. We remark that the existence of enough moments of $P_{0}$ would be sufficient. Moreover, $f$ may have local singularities.

Under the above assumptions, the potentials $U, V_{\text {per }}, V_{\omega}$ and their sums belong to $L_{\text {unif }}^{2 \text {, loc }}$, hence they are $H_{0}$-bounded (see [14], Theorem XIII.96). Moreover, the operators

$$
\begin{aligned}
& H_{0}:=-\frac{d^{2}}{d x^{2}} \quad(\text { the free Hamiltonian }) \\
& H_{U}:=H_{0}+U \\
& H_{\mathrm{per}}:=H_{0}+V_{\mathrm{per}}, \\
& H_{U, \mathrm{per}}:=H_{0}+U+V_{\mathrm{per}}
\end{aligned}
$$

and $H_{\omega}$ are essentially self-adjoint on $C_{0}^{\infty}(\mathbb{R})$.

Throughout this work, $\|\cdot\|$ will denote the $L^{2}$-norm. The spectrum and the essential spectrum of a linear operator $A$ will be denoted by $\sigma(A)$ and $\sigma_{\text {ess }}(A)$, respectively. 


\section{The essential spectra of $H_{U+V}$ and $H_{U \text {,per }}$}

One of the main observations of this section is the following result.

Theorem 2.1. Let $U_{1}, U_{2}, V: \mathbb{R} \rightarrow \mathbb{R}$ be $H_{0}$-bounded measurable functions and

$$
U_{j}(x) \underset{x \rightarrow-\infty}{\longrightarrow} a^{-}, \quad U_{j}(x) \underset{x \rightarrow \infty}{\longrightarrow} a^{+} \quad(j=1,2)
$$

for some $a^{ \pm} \in \mathbb{R}$. Then

$$
\sigma_{\mathrm{ess}}\left(H_{U_{1}+V}\right)=\sigma_{\mathrm{ess}}\left(H_{U_{2}+V}\right)
$$

Proof. We need to show that

$$
\begin{aligned}
& \sigma_{\mathrm{ess}}\left(H_{U_{1}+V}\right) \subset \sigma_{\mathrm{ess}}\left(H_{U_{2}+V}\right), \\
& \sigma_{\mathrm{ess}}\left(H_{U_{2}+V}\right) \subset \sigma_{\mathrm{ess}}\left(H_{U_{1}+V}\right) .
\end{aligned}
$$

We'll prove the first inclusion (the proof of the second one is similar). Let $\lambda \in \sigma_{\text {ess }}\left(H_{U_{1}+V}\right)$. By Weyl's criterion and Theorem 3.11 in [4] we conclude that there is a Weyl sequence of functions $\varphi_{n} \in C_{0}^{\infty}(\mathbb{R})(n \in \mathbb{N})$ such that

$$
\begin{gathered}
\left\|\varphi_{n}\right\|=1 \quad(n \in \mathbb{N}), \\
\left\|\left(H_{U_{1}+V}-\lambda I\right) \varphi_{n}\right\| \rightarrow 0
\end{gathered}
$$

and either

$$
\operatorname{supp} \varphi_{n} \subset(-\infty,-n) \quad \text { for all } n \in \mathbb{N}
$$

or

$$
\operatorname{supp} \varphi_{n} \subset(n, \infty) \text { for all } n \in \mathbb{N}
$$

holds. Assume (3) is true, then

$$
\begin{gathered}
\left\|\left(H_{U_{1}+V}-\lambda I\right) \varphi_{n}\right\|-\left\|\left(H_{V}-\left(\lambda-a^{-}\right) I\right) \varphi_{n}\right\| \rightarrow 0, \\
\left\|\left(H_{U_{2}+V}-\lambda I\right) \varphi_{n}\right\|-\left\|\left(H_{V}-\left(\lambda-a^{-}\right) I\right) \varphi_{n}\right\| \rightarrow 0
\end{gathered}
$$

and hence

$$
\left\|\left(H_{U_{1}+V}-\lambda I\right) \varphi_{n}\right\|-\left\|\left(H_{U_{2}+V}-\lambda I\right) \varphi_{n}\right\| \rightarrow 0 .
$$

From this and (2) we obtain

$$
\left\|\left(H_{U_{2}+V}-\lambda I\right) \varphi_{n}\right\| \rightarrow 0
$$

therefore $\lambda \in \sigma_{\text {ess }}\left(H_{U_{2}+V}\right)$.

As a corollary to the proof of Theorem 2.1 we get 
Corollary 2.2. Let $U, V: \mathbb{R} \rightarrow \mathbb{R}$ be measurable, $H_{0}$-bounded and

$$
U(x) \underset{x \rightarrow-\infty}{\longrightarrow} a^{-}, \quad U(x) \underset{x \rightarrow \infty}{\longrightarrow} a^{+}
$$

(in the usual sense), where $a^{ \pm} \in \mathbb{R}$. Then

$$
\sigma_{\mathrm{ess}}\left(H_{U+V}\right) \subset\left(a^{-}+\sigma_{\mathrm{ess}}\left(H_{V}\right)\right) \cup\left(a^{+}+\sigma_{\mathrm{ess}}\left(H_{V}\right)\right) .
$$

Remark 2.3. The previous theorem shows that the knowledge of $V$ and $a^{ \pm}$is sufficient for the unique determination of $\sigma_{\mathrm{ess}}\left(H_{U+V}\right)$. In fact,

$$
\sigma_{\mathrm{ess}}\left(H_{U+V}\right)=\sigma_{\mathrm{ess}}\left(H_{U_{c}+V}\right)
$$

where $U_{c}=a^{-} \chi_{(-\infty, 0]}+a^{+} \chi_{(0, \infty)}$.

In general, equality in (4) does not hold. However, for the case of periodic potentials we have:

Theorem 2.4. Let $U: \mathbb{R} \rightarrow \mathbb{R}$ be measurable, $H_{0}$-bounded and satisfy the conditions

$$
U(x) \underset{x \rightarrow-\infty}{\longrightarrow} a^{-}, \quad U(x) \underset{x \rightarrow \infty}{\longrightarrow} a^{+},
$$

and let $V_{\mathrm{per}}$ be a $H_{0}$-bounded periodic potential, then

$$
\sigma_{\mathrm{ess}}\left(H_{U, \mathrm{per}}\right)=\left(a^{-}+\sigma_{\mathrm{ess}}\left(H_{\mathrm{per}}\right)\right) \cup\left(a^{+}+\sigma_{\mathrm{ess}}\left(H_{\mathrm{per}}\right)\right) .
$$

Proof. In the view of Corollary 2.2, we need to prove that

$$
\begin{aligned}
& a^{-}+\sigma_{\text {ess }}\left(H_{\text {per }}\right) \subset \sigma_{\text {ess }}\left(H_{U, \text { per }}\right), \\
& a^{+}+\sigma_{\text {ess }}\left(H_{\text {per }}\right) \subset \sigma_{\text {ess }}\left(H_{U, \text { per }}\right) .
\end{aligned}
$$

We'll prove (6) (the proof of (7) is similar). Let $\lambda \in a^{-}+\sigma_{\text {ess }}\left(H_{\text {per }}\right)$, i.e., $\lambda-a^{-} \in \sigma_{\text {ess }}\left(H_{\text {per }}\right)$. Then there is a Weyl sequence $\varphi_{n} \in C_{0}^{\infty}(\mathbb{R})(n \in \mathbb{N})$ with

1. $\left\|\varphi_{n}\right\|=1 \quad(n \in \mathbb{N})$,

2. $\left\|\left(H_{\text {per }}-\left(\lambda-a^{-}\right) I\right) \varphi_{n}\right\| \rightarrow 0$,

Since $V_{\text {per }}$ is periodic, any shift of $\varphi_{n}$ by an integer is also a Weyl sequence for $H_{\text {per }}+a^{-}$. Thus we may assume that $\operatorname{supp} \varphi_{n} \subset(-\infty,-n)(n \in \mathbb{N})$. As in the previous proofs, one easily sees that this sequence is also a Weyl sequence for $H_{\text {per }}+U$.

Remark 2.5. It is well known that under the above assumptions on $V_{\text {per }}$ the equality $\sigma_{\text {ess }}\left(H_{\text {per }}\right)=\sigma\left(H_{\text {per }}\right)$ holds (see [6], [14]).

Remark 2.6. The special case of the formula (5) in which $V_{\text {per }}=0$, yields

$$
\sigma_{\mathrm{ess}}\left(H_{U}\right)=\left[\min \left\{a^{+}, a^{-}\right\}, \infty\right)
$$


This equality was obtained by I. Khachatryan and A. Petrosyan [9] under the condition

$$
\int_{-\infty}^{0}\left|U(x)-a^{-}\right| d x+\int_{0}^{\infty}\left|U(x)-a^{+}\right| d x<\infty
$$

(see also [3]).

\section{The essential spectrum of $H_{\omega}$}

We turn to the spectrum of $H_{\omega}$. To do so, we first describe the spectrum of $H_{\text {per }}+V_{\omega}$, i.e., the case $U=0$. We follow the investigation in [10].

Definition 3.1. A potential $W(x)=\sum_{k \in \mathbb{Z}} \rho_{k} f(x-k)$ is called admissible, if $\rho_{k} \in \operatorname{supp} P_{0}$ for all $k$. Let us denote by $\mathscr{P}$ the set of all admissible potentials, generated by $\ell$-periodic $\rho_{k}$ for some $\ell \in \mathbb{N}$.

Theorem 3.2. The spectrum $\sigma\left(H_{\mathrm{per}}+V_{\omega}\right)$ is almost surely independent of $\omega$ and is (almost surely) given by

$$
\sigma\left(H_{\mathrm{per}}+V_{\omega}\right)=\sigma_{\mathrm{ess}}\left(H_{\mathrm{per}}+V_{\omega}\right)=\overline{\bigcup_{W \in \mathscr{P}} \sigma\left(H_{\mathrm{per}}+W\right)}
$$

In the case of $V_{\text {per }}=0$ Theorem 3.2 is proved in [10]; the proof in the general case is similar.

In particular, the following result was proved in [10].

Lemma 3.3. If $W$ is a periodic admissible potential and $\lambda \in \sigma\left(H_{\mathrm{per}}+W\right)$, then there are sequences $\varphi_{n}^{+}, \varphi_{n}^{-} \in L^{2}(\mathbb{R})(n \in \mathbb{N})$ in the domain of $H_{\text {per }}+W$, such that

1. $\left\|\varphi_{n}^{+}\right\|=\left\|\varphi_{n}^{-}\right\|=1 \quad(n \in \mathbb{N})$.

2. The supports of $\varphi_{n}^{+}$and $\varphi_{n}^{-}$are compact and satisfy $\operatorname{supp} \varphi_{n}^{+} \subset[n, \infty) \quad$ and $\operatorname{supp} \varphi_{n}^{-} \subset(-\infty,-n] \quad(n \in \mathbb{N})$.

3. For almost all $\omega$

$$
\left\|\left(H_{\mathrm{per}}+V_{\omega}-\lambda\right) \varphi_{n}^{+}\right\| \rightarrow 0 \quad \text { and } \quad\left\|\left(H_{\mathrm{per}}+V_{\omega}-\lambda\right) \varphi_{n}^{-}\right\| \rightarrow 0 .
$$

From this we conclude

Theorem 3.4. Almost surely

$$
\sigma_{\mathrm{ess}}\left(H_{\mathrm{per}}+U+V_{\omega}\right)=\left(a^{-}+\sigma_{\mathrm{ess}}\left(H_{\mathrm{per}}+V_{\omega}\right)\right) \cup\left(a^{+}+\sigma_{\mathrm{ess}}\left(H_{\mathrm{per}}+V_{\omega}\right)\right) .
$$

Proof. By Corollary 2.2 we know that

$$
\sigma_{\mathrm{ess}}\left(H_{\mathrm{per}}+U+V_{\omega}\right) \subset\left(a^{-}+\sigma_{\mathrm{ess}}\left(H_{\mathrm{per}}+V_{\omega}\right)\right) \cup\left(a^{+}+\sigma_{\mathrm{ess}}\left(H_{\mathrm{per}}+V_{\omega}\right)\right) .
$$


To prove the converse we observe that for any $W \in \mathscr{P}$

$$
a^{ \pm}+\sigma\left(H_{\mathrm{per}}+W\right) \subset \sigma_{\mathrm{ess}}\left(H_{\mathrm{per}}+U+W\right)
$$

by Theorem 2.4. It is easy to see (e.g. as in [10]) that almost surely for $W \in \mathscr{P}$

$$
\sigma_{\mathrm{ess}}\left(H_{\mathrm{per}}+U+W\right) \subset \sigma_{\mathrm{ess}}\left(H_{\mathrm{per}}+U+V_{\omega}\right)
$$

Hence we conclude that

$$
\bigcup_{W \in \mathscr{P}} \sigma\left(H_{\mathrm{per}}+W+a^{+}\right) \cup \bigcup_{W \in \mathscr{P}} \sigma\left(H_{\mathrm{per}}+W+a^{-}\right) \subset \sigma_{\mathrm{ess}}\left(H_{\mathrm{per}}+U+V_{\omega}\right) .
$$

Since the right side is a closed set, we infer from Theorem 3.2 that almost surely

$$
\left(a^{-}+\sigma_{\mathrm{ess}}\left(H_{\mathrm{per}}+V_{\omega}\right)\right) \cup\left(a^{+}+\sigma_{\mathrm{ess}}\left(H_{\mathrm{per}}+V_{\omega}\right)\right) \subset \sigma_{\mathrm{ess}}\left(H_{\mathrm{per}}+U+V_{\omega}\right) .
$$

The following localization result is based on the work of D. Damanik and G. Stolz [5].

Theorem 3.5. Let $U$ be continuous, $V_{\text {per }}$ be bounded and $f$ satisfy the estimate

$$
c \chi_{I}(x) \leqslant f(x) \leqslant C \chi_{(0,1)} \quad(\text { a.e. } x \in \mathbb{R})
$$

with constants $0<c \leqslant C<\infty$ and a non-trivial subinterval I of $(0,1)$. Moreover, let

$$
\begin{array}{ll}
f(x)>0 & (\text { a.e. } x \in(a, b)), \\
f(x)=0 & (\text { a.e. } x \in \mathbb{R} \backslash(a, b))
\end{array}
$$

for a subinterval $(a, b) \subset(0,1)$. Then almost surely the operator $H_{\omega}$ has a dense point spectrum with exponentially decaying eigenfunctions and, possibly in addition, isolated eigenvalues with finite multiplicities.

Proof. Damanik and Stolz [5] proved that (under the formulated assumptions on $f$ ) if for a function $W_{0} \in L_{\mathbb{R}}^{\infty}(\mathbb{R})$ the set

$$
M\left(W_{0}\right):=\left\{\left.W_{0}(\cdot-n)\right|_{(0,1)}: n \in \mathbb{Z}\right\}
$$

is relatively compact in $L^{\infty}(0,1)$, then almost surely the operator $-\frac{d^{2}}{d x^{2}}+W_{0}+V_{\omega}$ has a dense point spectrum with exponentially decaying eigenfunctions and, possibly in addition, isolated eigenvalues with finite multiplicities. Since

$$
M\left(U+V_{\text {per }}\right)=M(U)+V_{\text {per }},
$$

hence it remains to show the relative compactness of $M(U)$. The continuity of $U$ and the existence of its finite limits at $\pm \infty$ imply the uniform continuity of $U$. The latter, in turn, implies the equicontinuity 
of $M(U)$. According to Arzela-Ascoli theorem, $M(U)$ is relatively compact in $C[0,1]$ and hence in $L^{\infty}(0,1)$.

\section{The Integrated Density of States}

In this section we investigate the integrated density of states of the operator $H_{\omega}$.

Definition 4.1. Let $A$ be a self-adjoint operator bounded below and with (possibly infinite) purely discrete spectrum $\lambda_{1}(A) \leqslant \lambda_{2}(A) \leqslant \lambda_{3}(A) \leqslant \ldots$, where the eigenvalues are counted according to their multiplicities. Denote

$$
N(A, E):=\#\left\{j: \lambda_{j}(A) \leqslant E\right\} \quad(E \in \mathbb{R}) .
$$

For $H=H_{0}+W$ with $W \in L_{\text {unif }}^{2, \text { loc }}$ and $a, b \in \mathbb{R}, a<b$ we define $H_{a, b}^{D}$ to be the operator $H$ restricted to $L^{2}(a, b)$ with Dirichlet boundary conditions both at $a$ and $b$. Similarly, $H_{a, b}^{N}$ has Neumann boundary conditions at $a$ and $b, H_{a, b}^{D, N}$ has Dirichlet boundary condition at $a$ and Neumann boundary condition at $b, H_{a, b}^{N, D}$ has Neumann boundary condition at $a$ and Dirichlet one at $b$.

If for $H=H_{0}+W$ the limit

$$
\mathscr{N}(E)=\mathscr{N}(H, E):=\lim _{L \rightarrow \infty} \frac{1}{2 L} N\left(H_{-L, L}^{D}, E\right)
$$

exists for all but countably many $E$, we call $\mathscr{N}(E)$ the integrated density of states for $H$.

It is well known that under our assumptions the integrated density of states for $H_{\text {per }}+V_{\omega}$ exists, more precisely:

Theorem 4.2. If $V_{\omega}$ satisfies the assumptions of Section 1, then the integrated density of states $\mathscr{N}\left(H_{\mathrm{per}}+V_{\omega}, E\right)$ almost surely exists and for all but countably many $E$ the following equalities hold:

$$
\mathscr{N}\left(H_{\mathrm{per}}+V_{\omega}, E\right)=\lim _{L \rightarrow \infty} \frac{N\left(H_{-L, L}^{N}(E)\right)}{2 L}=\lim _{L \rightarrow \infty} \frac{\mathbb{E}\left(N\left(H_{-L, L}^{D}(E)\right)\right)}{2 L}=\lim _{L \rightarrow \infty} \frac{\mathbb{E}\left(N\left(H_{-L, L}^{N}(E)\right)\right)}{2 L}
$$

( $\mathbb{E}$ denotes the expectation with respect to $\mathbb{P})$.

In the case of $V_{\text {per }}=0$ Theorem 4.2 is proved in [11]; the proof in the general case is similar and uses the method of Dirichlet-Neumann bracketing (see [14]). In particular, it is used:

Theorem 4.3. If $a<c<b$ and $X, Y \in\{D, N\}$, then

$$
N\left(H_{a, c}^{X, D}, E\right)+N\left(H_{c, b}^{D, Y}, E\right) \leqslant N\left(H_{a, b}^{X, Y}, E\right) \leqslant N\left(H_{a, c}^{X, N}, E\right)+N\left(H_{c, b}^{N, Y}, E\right) \quad(E \in \mathbb{R}) .
$$

For the integrated density of states of the operator $H_{\omega}$ we have the following result.

Theorem 4.4. The integrated density of states $\mathscr{N}\left(H_{\omega}, E\right)$ almost surely exists and can be expressed in terms of $\mathscr{N}_{1}(E)$, the integrated density of states of $H_{\mathrm{per}}+V_{\omega}$ by:

$$
\mathscr{N}\left(H_{\omega}, E\right)=\frac{1}{2} \mathscr{N}_{1}\left(E-a^{-}\right)+\frac{1}{2} \mathscr{N}_{1}\left(E-a^{+}\right) .
$$


To prove this result we need the following lemma:

Lemma 4.5. For the integrated density of states $\mathscr{N}_{1}$ of $H_{\mathrm{per}}+V_{\omega}$ we have

$$
\mathscr{N}_{1}(E)=\lim _{L \rightarrow \infty} \frac{1}{L} \mathbb{E}\left(N\left(\left(H_{\mathrm{per}}+V_{\omega}\right)_{M, L}^{X, Y}\right)\right)=\lim _{L \rightarrow \infty} \frac{1}{L} \mathbb{E}\left(N\left(\left(H_{\mathrm{per}}+V_{\omega}\right)_{-L,-M}^{X, Y}\right)\right)
$$

for any fixed $M \in \mathbb{R}$ and any $X, Y \in\{D, N\}$.

Proof. By the stationarity of the potential we have

$$
\mathbb{E}\left(N\left(\left(H_{\text {per }}+V_{\omega}\right)_{M, L}^{X, Y}\right)\right)=\mathbb{E}\left(N\left(\left(H_{\text {per }}+V_{\omega}\right)_{-(L-M) / 2,(L-M) / 2}^{X, Y}\right)\right) .
$$

Thus, the lemma follows from Theorem 4.2.

Now we prove Theorem 4.4.

Proof. For $L>|M|$ we have

$$
\begin{aligned}
\mathbb{E}\left(N\left(\left(H_{\omega}\right)_{-L, L}^{X, Y}\right)\right) \leqslant \mathbb{E}\left(N\left(\left(H_{\mathrm{per}}+U+V_{\omega}\right)_{-L,-M}^{X, N}\right)\right)+ \\
+\mathbb{E}\left(N\left(\left(H_{\omega}\right)_{-M, M}^{N, N}\right)\right)+\mathbb{E}\left(N\left(\left(H_{\mathrm{per}}+U+V_{\omega}\right)_{M, L}^{N, Y}\right)\right)
\end{aligned}
$$

We take $M>0$ so large that $\left|U(x)-a^{-}\right|<\varepsilon / 2$ for $x \leqslant-M$ and $\left|U(x)-a^{+}\right|<\varepsilon / 2$ for $x \leqslant M$. Let us divide inequality (8) by $2 L$. Then the middle term goes to zero as $L \rightarrow \infty$. Moreover, in the limit the first term on the right hand side can be bounded by $\frac{1}{2} \mathscr{N}_{1}\left(E-a^{-}\right)+\varepsilon / 2$. Similarly, the third term can be bounded by $\frac{1}{2} \mathscr{N}_{1}\left(E-a^{+}\right)+\varepsilon / 2$. Since $\varepsilon>0$ was arbitrary, we proved that

$$
\limsup _{L \rightarrow \infty} \mathbb{E}\left(N\left(\left(H_{\omega}\right)_{-L, L}^{X, Y}\right)\right) \leqslant \frac{1}{2} \mathscr{N}_{1}\left(E-a^{-}\right)+\frac{1}{2} \mathscr{N}_{1}\left(E-a^{+}\right) .
$$

The opposite inequality is proved using the analogue of (8) for Dirichlet boundary conditions (instead of Neumann ones).

\section{References}

[1] Aizenman M., Molchanov S., Localization at Large Disorder and at Extreme Energies: An Elementary Derivation. - Comm. Math. Phys., vol. 157 (1993), pp. 245-278, DOI: 10.1007/BF02099760

[2] Anderson P. W., Absence of diffusion in certain random lattices. - Phys. Rev. 109 (1958), pp. 1492-1505, DOI: 10.1103/PhysRev.109.1492

[3] Asatryan H. A., Inverse scattering problem for Sturm-Liouville operators. - Journal of Contemporary Mathematical Analysis, vol. 40, No 2 (2005), pp. 14-27

[4] Cycon H. L., Froese R. G., Kirsch W., Simon B., Schrödinger Operators. - Springer, 2008 
[5] Damanik D., Stolz G., A continuum version of the Kunz-Souillard approach to localization in one dimension. - J. reine angew. Math. 660 (2011), pp. 99-130, DOI: 10.1515/CRELLE.2011.070

[6] Eastham M. S. P., The Spectral Theory of Periodic Differential Equations. - Belfast, Scottish Academic Press, 1973

[7] Fröhlich J., Spencer T., Absence of diffusion in the Anderson tight binding model for large disorder or low energy. - Comm. Math. Phys., vol. 88 (1983), pp. 151-184, DOI: 10.1007/BF01209475

[8] Goldsheid I. Ya., Molchanov S. A., Pastur L. A., A Pure Point Spectrum of the Stochastic OneDimensional Schrödinger Operator. - Func. Anal. Its Appl., vol. 11, No 1 (1977), pp. 1-8, DOI: $10.1007 / \mathrm{BF} 01135526$

[9] Khachatryan I. G., Petrosyan A. H., About the eigenfunction expansion associated with a selfadjoint differential operator with coefficients having certain behavior at infinity (in Russian). Proceedings of Yerevan State University, Natural Sciences, No 1 (2004), pp. 22-27

[10] Kirsch W., Martinelli F., On the spectrum of Schrödinger operators with a random potential. Comm. Math. Phys. 85, No 3 (1982), pp. 329-350, DOI: 10.1007/BF01208718

[11] Kirsch W., Martinelli F., On the density of states of Schrödinger operators with a random potential. - J. Phys. A: Math. Gen., vol. 15, No 7 (1982), pp. 2139-2156, DOI: $10.1088 / 0305-4470 / 15 / 7 / 025$

[12] Kunz H., Souillard B., Sur le spectre des operateurs aux differences finies aleatoires. - Comm. Math. Phys., vol. 78 (1980), pp. 201-246, DOI: 10.1007/BF01942371

[13] Pastur L. A., Spectra Of Random Self Adjoint Operators. - Russ. Math. Surv., vol. 28, No 1 (1973), pp. 1-67, DOI: 10.1070/RM1973v028n01ABEH001396

[14] Reed M., Simon B., Methods of Modern Mathematical Physics, vol. 4. Analysis of Operators. Academic Press, 1978 